\title{
Tecnologia Educacional sobre limpeza e desinfecção de brinquedos para ambientes escolares frente à pandemia da COVID-19
}

\author{
Educational technology on cleaning and disinfecting toys for school environments in the face of the \\ COVID-19 pandemic \\ Tecnología educativa en limpieza y desinfección de juguetes para ambientes escolares ante la \\ pandemia COVID-19
}

\begin{abstract}
Patrícia Pinto Braga ${ }^{1}$ (C)
Márcia Christina Caetano Romano ${ }^{1}$ (1) Elaine Cristina Rodrigues Gesteira ${ }^{1}$ (D) Dayse Bazílio Rosa de Souza ${ }^{1}$ (i) Marina Guedes Pinto ${ }^{1}$ (1) Verônica Gomes dos Santos ${ }^{1}$ [D
\end{abstract}

1. Universidade Federal de São João Del Rei, Campus Centro Oeste, Curso de Enfermagem. Divinópolis, MG, Brasil.
Autor correspondente:

Patrícia Pinto Braga.

E-mail: patriciabragaufsj@gmail.com.

Recebido em 26/01/2021.

Aprovado em 28/04/2021.

DOI:https://doi.org/10.1590/2177-9465-EAN-2021-0023

\section{RESUMO}

Objetivo: descrever a construção e validação do conteúdo e aparência de tecnologia educacional sobre limpeza e desinfecção de brinquedos para ambientes escolares e creches, no contexto de pandemia do coronavírus. Método: Pesquisa metodológica de validação, orientada pela teoria da Psicometria. Realizada em três fases: (1) revisão bibliográfica (2) elaboração da tecnologia educacional (3) avaliação, aperfeiçoamento e validação do conteúdo e aparência da cartilha. Participaram 20 juízes e 16 profissionais da educação infantil. Para garantir a validação, consideramos o índice de validade de conteúdo de, no mínimo, 0,8. Os dados foram tratados no software Statistical Package for Social Science e submetidos à análise descritiva de distribuição de frequências. Resultados: $O$ índice de validade de conteúdo global foi de 0,99 para juízes e 0,96 para o público-alvo. Os domínios da cartilha apresentaram índice de validade de conteúdo superior a 0,8 sugerindo que é um material representativo, quanto à aparência e conteúdo. Conclusão e implicações para prática: A cartilha digital pode ser considerada, no contexto das atividades educativas em enfermagem, instrumento capaz de favorecer orientações claras e confiáveis sobre a limpeza e desinfecção de brinquedos, para ambientes escolares, no cenário de pandemia do novo coronavírus.

Palavras-Chave: Promoção da Saúde Escolar; Jogos e brinquedos; Tecnologia Educacional; Estudo de Validação; Infecções por Coronavírus.

\section{ABSTRACT}

Objective: to describe the construction and validation, of content and appearance, of an educational technology on cleaning and disinfecting toys for school environments and daycare centers in the context of the coronavirus pandemic. Method: Validation methodological research, guided by the Psychometry theory. Three phases were carried out, namely: (1) bibliographic review, (2) elaboration of the educational technology, and (3) evaluation, improvement and validation of the content and appearance of the booklet. The participants were 20 evaluators and 16 professionals specialized in early childhood education. To ensure validation, we consider a Content Validity Index of at least 0.8. The data were treated using the Statistical Package for Social Science software and submitted to descriptive analysis of frequency distribution. Results: The global improvement and validation of the content was 0.99 for the evaluators and 0.96 for the target audience. The domains of the booklet showed improvement and content validation above 0.8 , suggesting that this is a representative material, in terms of appearance and content. Conclusion and implications for the practice: In the context of educational activities in Nursing, the digital booklet can be considered as an instrument capable of favoring clear and reliable guidance on cleaning and disinfecting toys for school environments in the coronavirus pandemic scenario.

Keywords: School Health Services; Play and Playthings; Educational Technology; Validation Study; Coronavirus Infections.

\section{RESUMEN}

Objetivo: describir la construcción y validación, en términos de contenido y apariencia, de tecnología educativa sobre la limpieza y desinfección de juguetes para ambientes escolares y guarderías en el contexto de la pandemia por coronavirus. Método: investigación metodológica de validación, guiada por la teoría de la Psicometría. Se realizó en tres etapas, a saber (1) revisión bibliográfica, (2) elaboración de la tecnología educativa, y (3) evaluación, mejora y validación del contenido y la apariencia del folleto. Participaron 20 jueces y 16 profesionales de educación infantil. Para garantizar la validación, consideramos un Índice de Validez de Contenido mínimo de 0,8. Los datos se trataron en el software Statistical Package for Social Science y fueron sometidos a análisis descriptivo de distribución de frecuencias. Resultados: El Índice de Calidez de Contenido global fue de 0,99 para los jueces y de 0,96 para el público objetivo. Los dominios del cuadernillo presentaron un Índice de Validez de Contenido superior a 0.8 , lo que sugiere que se trata de un material representativo, en términos de apariencia y contenido. Conclusión e implicaciones para la práctica: En el contexto de las actividades educativas en enfermería, el folleto digital puede considerarse como instrumento capaz de favorecer una orientación clara y confiable sobre la limpieza y desinfección de juguetes para ambientes escolares en el escenario pandémico del coronavirus.

Palabras clave: Servicios de Salud Escolar; Juego e Implementos de Juego; Tecnología Educativa; Estudio de Validación; Infecciones por Coronavirus. 


\section{INTRODUÇÃO}

O atual cenário mundial de pandemia associado à Doença do Coronavírus 2019 (COVID-19) ${ }^{1}$ que possui como agente etiológico o SARS-CoV-2 (Severe Acute Respiratory Syndrome(Coronavirus) $)^{2}$ afeta a todos e, ainda, não sabemos quais serão as mudanças em longo prazo. Assim, medidas preventivas à contaminação têm se tornado urgentes e necessárias. Vários países como o Brasil adotaram medidas para desacelerar a transmissão da COVID-19, como o distanciamento físico, uso de máscaras faciais, higienização frequente das mãos e dos ambientes e superfícies frequentemente tocadas e, até mesmo, a suspensão das atividades escolares presenciais ${ }^{3}$.

Existem, relativamente, poucos casos de COVID-19 em crianças, quando comparados aos da população geral, representando de 1 a $5 \%$ dos casos diagnosticados, contudo o grupo infantil pode desenvolver COVID-19 grave, e um número não desprezível de casos pode apresentar uma condição inflamatória multissistêmica muito grave com algumas características semelhantes às da doença de Kawasaki e síndrome do choque tóxico ${ }^{4-6}$.

No Brasil, até o dia primeiro de março de 2021, 8774 crianças até cinco anos de idade foram hospitalizadas em função de Síndrome Respiratória Aguda Grave por Covid-19, e destas, 627 foram a óbito. A mortalidade foi maior entre crianças de até um ano de vida, perfazendo um total de 420 vidas perdidas ${ }^{7,8}$. Esse cenário demonstra a magnitude do problema na população infantil.

Assim, reconhecemos que investir em estratégias educativas, para profissionais da educação infantil e do ensino fundamental, como forma de prevenção ao Coronavírus torna-se relevante, pois poderá desacelerar a transmissão do vírus contribuindo para que os sistemas de saúde locais possam assistir dentro de sua capacidade e dar tempo para a produção de evidências científicas consistentes sobre a cura e/ou erradicação dessa doença ${ }^{9}$.

Neste estudo, partimos da premissa que, dentre as diferentes formas de prevenção da COVID-19, está a produção e utilização de material educativo confiável sobre a temática limpeza e desinfecção de brinquedos. Limpar e desinfectar brinquedos tem sido recomendado como forma de diminuição da disseminação e prevenção de contaminação pelo Coronavírus por ser um objeto ou superfície frequentemente tocada e potencialmente contaminada $^{10,11}$. É notório que, especialmente em ambientes escolares e creches, brinquedos são objetos muito manipulados e compartilhados pelas crianças, e podem ser levados à boca e ficar em contato direto com o chão ou outras superfícies sujas e contaminadas.

Revisão sistemática sobre a persistência do novo Coronavírus em superfícies inanimadas revela que o SARS-CoV-2 pode persistir em metal, vidro ou plástico por até nove dias e pode ser inativado eficientemente por procedimentos de desinfecção utilizando, por exemplo, etanol (62 a 70\%), peróxido de hidrogênio a $0,5 \%$ ou hipoclorito de sódio a $0,1 \%{ }^{10}$. Dados ainda mais recentes apontam sua sobrevivência por até dezessete dias em superfícies o que contribui para os contágios e a disseminação acelerada desse patógeno ${ }^{12}$.

Não encontramos estudos específicos sobre o SARSCov-2 em objetos como brinquedos, contudo há evidências de que os brinquedos são as formas mais comuns de promover a disseminação de microrganismos entre crianças, especialmente quando está associada à lavagem inadequada das mãos ${ }^{13-15}$. Estudos que analisaram a presença de microorganismos em diferentes tipos de brinquedos como metal, plástico, tecido e outros apontam esses objetos como reservatórios de microorganismos causadores de infecções ${ }^{16-18}$. Como brinquedos podem contribuir com a disseminação de doenças, é recomendável que haja uma rotina de sua limpeza e desinfecção, porém observa-se que, na prática, pouca atenção é dada a esse assunto ${ }^{16,18}$.

Identificamos em nosso cotidiano um discurso que, em ambientes escolares, há riscos de uma disseminação acelerada do novo Coronavírus. Esse discurso vem apoiando gestores do Brasil e do mundo na decisão pela suspensão das atividades presenciais, contudo os prejuízos sociais são imensuráveis. Nesse sentido, a tecnologia educacional aqui produzida, embora não responda a todas as demandas da educação infantil para prevenção da COVID-19, contribui com informações pertinentes e confiáveis que podem ser utilizadas por profissionais de enfermagem e da educação em ações educativas, com vistas à promoção da saúde escolar.

O material educativo produzido para validação nesta pesquisa refere-se a orientações relativas à limpeza e desinfecção dos diferentes tipos de brinquedos de uso pedagógico em espaços como escolas e creches. Reconhecemos que materiais educativos devem ser confiáveis, acessíveis, claros, significativos e adequados à realidade do leitor. Enfermeiros devem produzir material educativo de forma adequadamente planejada, realizando a avaliação e validação do material para atender às reais demandas do contexto ${ }^{19}$.

Nesta pesquisa partimos da compreensão de que a educação em saúde é um processo que deve propiciar reflexões e permitir uma aprendizagem significativa, favorecendo mudanças de atitudes e construção de autonomia, diante de uma realidade que precisa ser transformada ${ }^{20}$. $\mathrm{O}$ material educativo é um recurso para a educação em saúde na medida em que pode apoiar processos de ensino e aprendizagem. E há evidência de que seu uso tem se mostrado relevante na prática de cuidados, contribuindo com o acesso ao conhecimento. Nesse contexto, o enfermeiro poderá utilizá-lo para desenvolver atitudes e habilidades, em um público-alvo, com vistas a prevenir doenças e promover a saúde ${ }^{19}$.

Não há consenso na literatura sobre o método de higienização de brinquedos em ambientes escolares ou de serviços de saúde ${ }^{21}$ o que reforça a relevância deste estudo. Reconhecemos que, em um contexto de ausência de consenso e escassez de evidências, é oportuna a validação por peritos e público-alvo sobre o conteúdo do material educativo para viabilizar a divulgação de orientações confiáveis. 
Considerando o exposto, objetivou-se descrever a construção e validação do conteúdo e aparência de tecnologia educacional sobre limpeza e desinfecção de brinquedos para ambientes escolares e creches no contexto de pandemia do coronavírus.

\section{MÉTODO}

Pesquisa metodológica, de caráter descritivo e abordagem quantitativa, para desenvolvimento, avaliação, aperfeiçoamento e validação de um material educativo. Estudos dessa natureza são do tipo não experimental e o foco é na elaboração de novos instrumentos confiáveis, para a prática em enferamgem ${ }^{22}$.

Previamente, ao início da pesquisa, este estudo foi aprovado pelo Comitê de Ética em Pesquisa da Universidade Federal de São João del Rei (UFSJ), conforme parecer 31748520.8.0000.5545. Todo o processo de pesquisa e utilização dos dados seguiu os termos da Resolução no 466 de 12 de dezembro de 2012, do Conselho Nacional de Saúde, sobre pesquisa envolvendo seres humanos.

Este estudo de validação foi realizado, considerando três fases: Fase 1: Revisão bibliográfica sobre limpeza e desinfecção de brinquedos e as formas transmissão, disseminação e prevenção do novo Coronavírus; Fase 2: Elaboração da Cartilha; Fase 3: Avaliação, aperfeiçoamento e validação do conteúdo e aparência da cartilha pelos juízes e público-alvo, para produção da versão final da cartilha.

Para a elaboração da cartilha digital, buscamos informações teóricas e metodológicas sobre a produção de material educativo na área da saúde. Portanto, seguimos as seguintes etapas: (1) definição do tema; (2) pesquisa bibliográfica; (3) elaboração do roteiro, capítulos e conteúdo; (4) desenvolvimento da cartilha; (5) Versão da cartilha digital para validação ${ }^{19}$.

Para a revisão, realizamos a busca de artigos científicos, completos e disponíveis de forma online, publicados nos últimos cinco anos (2015 - 2020), em português, inglês e espanhol utilizando os descritores "Promoção da Saúde Escolar", "Jogos e Brinquedos", "Tecnologia Educacional", "Estudo de Validação" e "Infecções por Coronavírus", utilizando o operador boleano AND. A busca foi feita em abril de 2020, nas seguintes bases de dados: Literatura Latino-Americana e do Caribe em Ciências da Saúde (Lilacs) (5 publicações), Biblioteca Virtual de Saúde (BVS), (6 publicações), e Literatura Internacional em Ciências da Saúde (Medline) (23 publicações). Associado a isso, foram feitas buscas de Manuais Técnicos, Normas e Resoluções em Sites de Instituições Especializadas que produzem material de interesse a saber: Ministério da Saúde, Sociedade Brasileira de Pediatria, Sociedade Brasileira de Infectologia, Centro de Controle e Prevenção de Doenças dos Estados Unidos (CDC), Agência Nacional de Vigilância Sanitária (ANVISA) e Organização Mundial da Saúde (OMS). Após leitura do material, 19 produções subsidiaram a construção da cartilha.

Para alcançar o público-alvo, a cartilha digital será amplamente divulgada e ficará disponível para acesso, a partir de um link, em diferentes mídias sociais e em um blog organizado pelas pesquisadoras deste estudo. Faremos também a divulgação por correio eletrônico, enviando o material para secretarias municipais de Educação e para a rede de pesquisadores dos autores deste artigo.

Como método de validação, adotamos a teoria da Psicometria ${ }^{23}$ que está ancorada em três polos: teórico, experimental e analítico. O polo teórico possui foco na fundamentação prévia e validação do construto e é útil para a realização de pesquisas que se ocupam da construção e validação de ferramentas ${ }^{21}$. Neste estudo, apenas os procedimentos do polo teórico foram abordados, considerando que o objeto desta pesquisa foi um material educativo, uma cartilha digital.

A população do estudo constituiu-se de dois grupos, os juízes, docentes pesquisadores da instituição proponente e os profissionais da educação, o público-alvo. No que se refere ao grupo de juízes, a população elegível foi composta por 150 docentes pesquisadores dos cursos de farmácia, medicina, enfermagem e bioquímica. Visando à inclusão no estudo, aplicou-se, por meio de consulta no currículo lattes, o sistema de pontuação de especialistas do Modelo de Fehring ${ }^{24}$, que atribui pontos conforme titulação, especialização, produção científica, conhecimento e tempo de atuação com a temática em discussão. Um total de 45 pesquisadores obtiveram pontuação mínima de cinco pontos. Pelo modelo, considerados aptos e, portanto, foram convidados a participar. Destes, 20 aceitaram fazer parte da investigação.

Quanto ao público-alvo, a população elegível foi composta por um profissional da educação de cada escola pública de educação infantil e de ensino fundamental do município da instituição proponente, perfazendo um total de 50 educadores. Como critério de inclusão destes profissionais, estabeleceu-se experiência de, no mínimo, um ano na educação infantil e/ou ensino fundamental. Após o convite, 16 profissionais concordaram em participar do estudo, constituindo o público-alvo.

Cabe destacar que é necessário o mínimo de nove juízes ${ }^{25}$ para promover a validação de um material educativo. Portanto, a amostra obtida de participantes de ambos os grupos foi considerada suficiente.

A cartilha foi submetida à avaliação dos juízes e do públicoalvo. Para a realização da coleta de dados, que ocorreu entre os meses de junho a agosto de 2020 , foram utilizados dois instrumentos objetivos elaborados pelos autores e aplicados, através de correio eletrônico. Os juízes responderam ao formulário, contendo perguntas sobre os aspectos técnicos do conteúdo da cartilha, como coerência, efetividade, capacidade, potencialidade, texto, ortografia e estética. O formulário dos profissionais da educação continha perguntas acerca do conteúdo da cartilha e elementos relacionados à capacidade educativa em saúde. Dessa forma, foi possível a obtenção de medidas objetivas, ou seja, foi possível quantificar a opinião dos participantes em relação à cartilha. Ao final de cada questão os participantes poderiam justificar suas respostas e/ou dar sugestões referentes ao material educativo.

Os dados coletados foram tabulados no Programa Microsoft Excel 2003 e observou-se a consistência das informações. Para a análise, utilizou-se o software Statistical Package for Social 
Science versão 23.0 (SPSS Inc., Chicago, IL.) Foi realizada análise descritiva de todas as variáveis investigadas mediante a distribuição de frequências.

Utilizou-se o Índice de Validade de Conteúdo (IVC) para medir a concordância dos especialistas quanto aos itens avaliados. O cálculo considerou a soma do número de juízes ou público-alvo que avaliaram o item como totalmente adequado + adequado + parcialmente adequado dividido pelo número de juízes ou do público-alvo ${ }^{25}$. Visando avaliar a cartilha de forma ampla e como um todo, calculou-se o Índice de Validade de Conteúdo Global, somando-se todos os IVC calculados, separadamente, e dividindo pelo número de itens do instrumento ${ }^{26}$.

Para determinar a adequação e pertinência de cada item abordado no instrumento de avaliação, foi considerado o IVC de, no mínimo, 0,827 para juízes e público-alvo. As sugestões e recomendações dos participantes foram analisadas a partir da literatura científica e aquelas, que foram pertinentes ao objetivo da cartilha, incorporadas na versão final.

\section{RESULTADOS}

\section{Descrição da elaboração e constituição da cartilha digital}

A revisão da literatura permitiu a elaboração de um roteiro e dos capítulos com o conteúdo da cartilha. Para desenvolver a arte e o design, utilizamos o programa Canva ${ }^{\circledR 28}$ uma plataforma de design gráfico que permite a criação de mídias, infográficos, pôsteres e outros conteúdos visuais de forma totalmente online, utilizando texto e imagens que compuseram a versão que foi apresentada aos participantes. Dentre os aspectos positivos dessa ferramenta, está o acesso livre, a possibilidade de diferentes pesquisadores trabalharem simultaneamente na revisão e edição do material e a variedade de layouts e figuras favorecendo a produção de um material visualmente atrativo. Participaram da edição da cartilha, utilizando o Canva ${ }^{\circledR 28}$, quatro pesquisadores autores deste estudo.

Durante a edição da cartilha, usando o Canva ${ }^{\circledR 28}$, o modelo de template adotado foi o Teal and Pink Animals Daycare Flyer, a paleta de cores usada foi branco, preto, verde, amarelo, vermelho, azul e rosa, o tamanho da fonte foi de 21 para títulos e 16 para o corpo do texto. As ilustrações usadas estavam disponíveis gratuitamente no Canva ${ }^{\circledR 28}$ e foram encontradas pesquisando os termos "brinquedos" e "estrelas". Utilizamos também imagens do Google, disponíveis gratuitamente e de domínio público, por meio de pesquisa, utilizando os termos "brinquedos de madeira", "ilustração crianças", "ilustração lavagem de mãos", "ilustração brinquedos de madeira" e "ilustração produtos de limpeza".

No material educativo, adotamos as definições do Centers for Disiase Control and Prevention (CDC) para limpeza e desinfecção, para o qual a limpeza é definida como a remoção de germes, sujeira e impurezas de superfícies ou objetos e funciona usando sabão (ou detergente) e água para remover fisicamente os germes das superfícies e a desinfecção é compreendida como a destruição de germes em superfícies ou objetos e funciona usando produtos químicos (desinfetantes) ${ }^{29}$.

A cartilha final intitulada Aprendizagem e diversão com segurança: limpeza e desinfecção de brinquedos em escolas e creches no contexto de pandemia do novo coronavírus, tem dimensão de 210x297mm e apresenta 26 páginas, é constituída por componentes pré-textuais (capa, contracapa, ficha catalográfica, sumário e apresentação), textuais (capítulos sobre o Coronavírus, formas de transmissão, contágio e prevenção, por que limpar e desinfetar brinquedos e qual a diferença entre limpeza e desinfecção, descrição detalhada de como limpar e desinfectar os diferentes tipos de brinquedo - plástico, borracha, madeira, metal, pelúcia, pano, papelão) e pós-textuais (dicas importantes, teste do conhecimento, referências bibliográficas). Na Figura 1, são apresentadas algumas páginas da versão final da cartilha.

Para situações que podem envolver a necessidade de impressão do material educativo, recomendamos que seja feita impressão em papel tamanho $\mathrm{A} 4$, considerando as dimensões da cartilha.

\section{Validação de conteúdo e aparência}

Contribuíram com a avaliação da cartilha 36 participantes e identificamos que a maioria são do sexo feminino $(80 \%$ dos juízes e $100 \%$ do público-alvo) e com mais de cinco anos de atuação no ensino universitário $(80 \%)$ ou educação básica (100\%). Dentre os juízes que participaram, $75 \%$ (n 15) possuem graduação em enfermagem e $90 \%$ possuem título de doutor. Apesar do número expressivo de enfermeiros respondentes, a pesquisa contou com a participação de pesquisadores com formação em outras áreas como psicologia (n 1), medicina ( $n 2$ ), arte (n 1) e farmácia (n 1).

A avaliação dos juízes quanto à coerência da cartilha com as necessidades e etapas de limpeza e desinfecção, sua utilidade e efetividade no processo educativo em saúde, capacidade de ser utilizada como tecnologia educacional e de estar em conformidade com o nível sociocultural do públicoalvo, obtiveram IVC de 1,0. A capacidade para promoção de mudanças e atitudes obteve IVC de 0,9. Esses achados são apresentados na Tabela 1.

A avaliação dos juízes sobre os aspectos textuais (clareza e cientificidade das informações) concordância, ortografia e aparência (ilustrações, estética, quantidade de páginas, tamanho de títulos e tópicos) obtiveram IVC de 1,0. Sobre a sequência lógica do conteúdo disposto na cartilha a concordância foi de 0,9 . Essas informações são apresentadas na Tabela 2.

A avaliação pelo público-alvo acerca da sequência, organização, clareza da escrita e abordagem dos assuntos necessários e aparência (pertinência e adequação das ilustrações) obteve IVC de 1,0. Acerca da capacidade da cartilha em gerar interesse e motivar a leitura foi obtida concordância de 0,8 . Todos os participantes pertencentes ao grupo do público-alvo concordaram que o material educativo possui a capacidade de promover atitude e reflexão sobre o tema (IVC $=1,0)$. Sobre a 

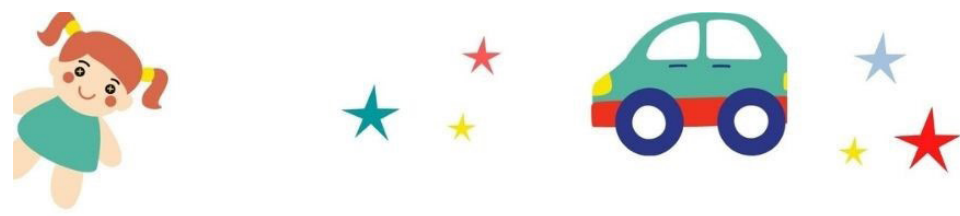

\section{S UM Á R I O}

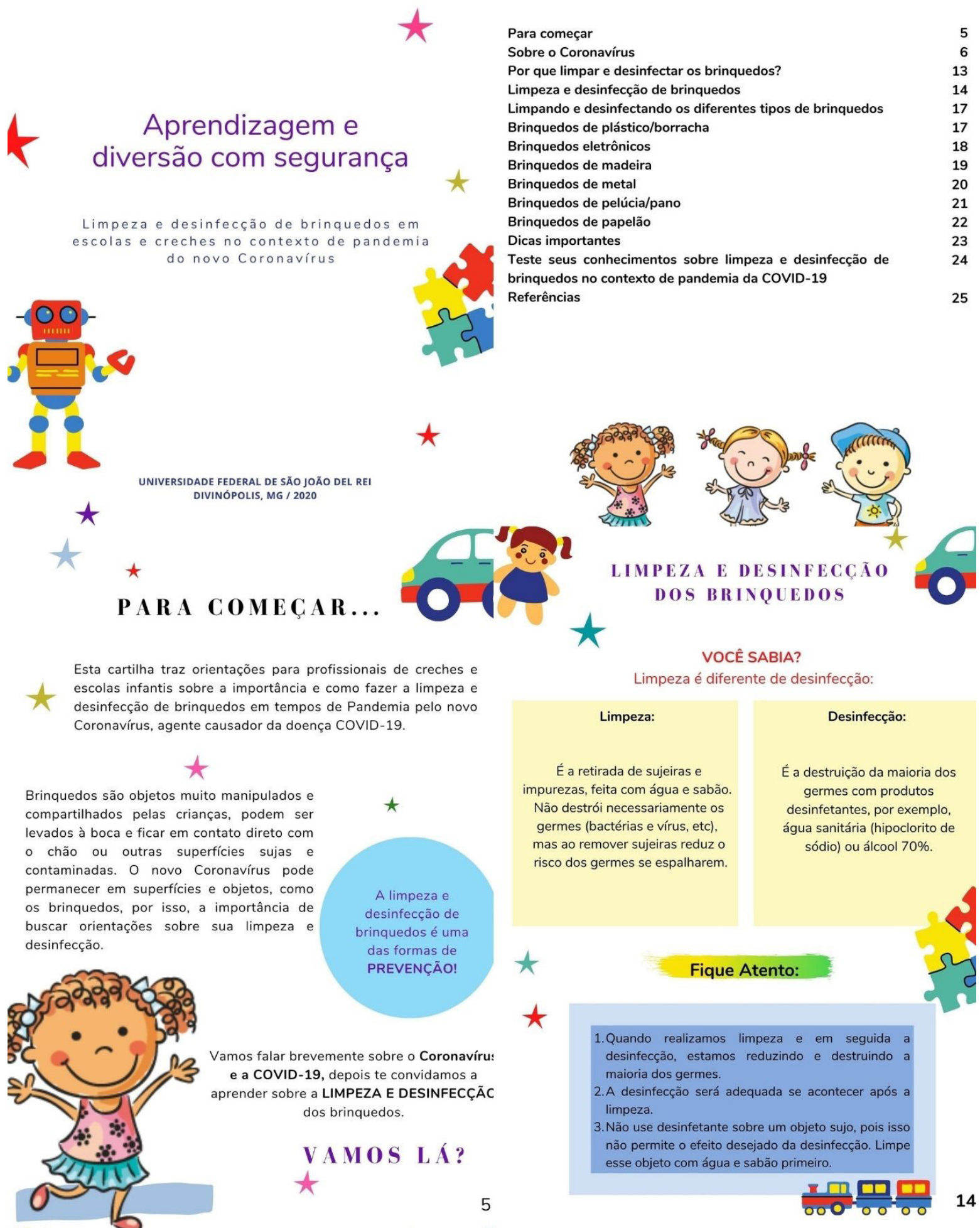

Figura 1. Algumas Páginas da Versão Final da Cartilha.

Fonte: Páginas da Cartilha, 2020. 
Tabela 1. Avaliação dos juízes acerca do conteúdo da cartilha. Divinópolis, MG, Brasil, 2020. N=20

\begin{tabular}{|c|c|c|c|c|}
\hline Item avaliado & $\begin{array}{c}\text { Totalmente } \\
\text { adequado }\end{array}$ & Adequado & $\begin{array}{c}\text { Parcialmente } \\
\text { adequado }\end{array}$ & IVC* \\
\hline Coerência com as necessidades de limpeza e desinfecção & 16 & 2 & 2 & 1,0 \\
\hline Coerência com a utilidade no processo educativo em saúde & 16 & 3 & 1 & 1,0 \\
\hline Efetividade para uso por adultos & 12 & 6 & 2 & 1,0 \\
\hline Capacidade de conformidade com nível sociocultural & 15 & 4 & 1 & 1,0 \\
\hline Capacidade para promoção de mudanças e atitudes** & 7 & 9 & 3 & 0,9 \\
\hline Capacidade de retratar temas essenciais à limpeza e desinfecção & 8 & 1 & 1 & 1,0 \\
\hline
\end{tabular}

Fonte: Dados da pesquisa, 2020

* IVC = Índice de Validade do Conteúdo ** Um especialista não respondeu

Tabela 2. Avaliação dos juízes sobre a cartilha quanto a aspectos textuais, ortográficos e estéticos. Divinópolis, MG, Brasil, 2020. $\mathrm{N}=20$

\begin{tabular}{|c|c|c|c|c|}
\hline Item avaliado & $\begin{array}{c}\text { Totalmente } \\
\text { adequado }\end{array}$ & Adequado & $\begin{array}{c}\text { Parcialmente } \\
\text { adequado }\end{array}$ & IVC* \\
\hline Informações claras e objetivas & 9 & 3 & 1 & 1,0 \\
\hline Informações da capa, contracapa, sumário e apresentação, coerentes & 16 & 4 & 0 & 1,0 \\
\hline Informações estruturadas em concordância e ortografia & 17 & 2 & 1 & 1,0 \\
\hline Concordância verbal e ortografia & 17 & 2 & 1 & 1,0 \\
\hline Adequação do tamanho do título e dos tópicos & 13 & 5 & 2 & 1,0 \\
\hline Ilustrações adequadas & 16 & 3 & 1 & 1,0 \\
\hline Quantidade de páginas adequada & 12 & 8 & 0 & 1,0 \\
\hline
\end{tabular}

Fonte: Dados da pesquisa, 2020

* IVC = Índice de Validade do Conteúdo ** Um especialista considerou inadequado

capacidade da cartilha em chamar atenção o IVC foi de 0,9. Esses resultados são apresentados na Tabela 3.

Os resultados referentes ao IVC global mostraram um elevado nível de concordância, ou seja, 0,99 para juízes e 0,96 para o público-alvo. Ainda assim, submetemos as sugestões destes à análise, a partir de evidências científicas, e incorporamos aquelas que estavam dentro do objetivo do material proposto e que poderiam contribuir com a qualidade da cartilha. No Quadro 1, apresentamos os ajustes e correções que foram incorporados no material educativo, bem como apresentamos as opiniões dos participantes da pesquisa, agrupadas por núcleos de sentido, acerca do material produzido.

\section{DISCUSSÃO}

Em relação ao processo de validação, consideramos que a diversidade profissional dos juízes e a titulação destes (90\% doutores) aliada aos conhecimentos do público-alvo, foram aspectos positivos, visto que diferentes saberes especializados, dentro da temática abordada, são relevantes em estudos de validação de tecnologias educacionais ${ }^{30,31}$. Soma-se a isso que uma abordagem multidisciplinar pode contribuir de maneira mais apropriada com estratégias de enfrentamento e prevenção da COVID-19 no atual cenário ${ }^{32}$.

A análise evidenciou elevado IVC global, tanto na avaliação pelos juízes de 0,99 como do público-alvo $(0,96)$, sugerindo que 
Tabela 3. Avaliação do público-alvo sobre a cartilha quanto ao conteúdo e aparência. Divinópolis, MG, Brasil, 2020. N=16

\begin{tabular}{|c|c|c|c|c|}
\hline Item avaliado & $\begin{array}{l}\text { Totalmente } \\
\text { adequado }\end{array}$ & $\begin{array}{l}\text { Parcialmente } \\
\text { adequado }\end{array}$ & Inadequado & IVC* \\
\hline Sequência do conteúdo & 16 & 0 & 0 & 1,0 \\
\hline Clareza das frases $* *$ & 15 & 0 & 0 & 1,0 \\
\hline Clareza da escrita & 16 & 0 & 0 & 1,0 \\
\hline $\begin{array}{l}\text { Abordagem de assuntos necessários à limpeza e desinfecção de } \\
\text { brinquedos }\end{array}$ & 16 & 0 & 0 & 1,0 \\
\hline Pertinência e adequação das ilustrações & 16 & 0 & 0 & 1,0 \\
\hline Pertinência das ilustrações complementarem o texto & 16 & 0 & 0 & 1,0 \\
\hline $\begin{array}{l}\text { Capacidade da cartilha de promover atitude e reflexão sobre o } \\
\text { tema }\end{array}$ & 16 & 0 & 0 & 1,0 \\
\hline Capacidade da capa de chamar a atenção**** & 15 & 0 & 0 & 0,9 \\
\hline Capacidade da cartilha de motivar o leitor até o final*** & 14 & 0 & 0 & 0,8 \\
\hline $\begin{array}{l}\text { Capacidade da cartilha de promover atitude e reflexão sobre o } \\
\text { tema }\end{array}$ & 16 & 0 & 0 & 1,0 \\
\hline
\end{tabular}

Fonte: Dados da pesquisa, 2020

* IVC = Índice de Validade do Conteúdo ** Um participante não soube responder *** Dois participantes não souberam responder **** Um participante não soube responder

é um material representativo, quanto à aparência e conteúdo. A etapa de validação pelos juízes é fundamental para identificar incoerências que possam prejudicar seu conteúdo ou compreensão e favorece maior rigor metodológico na construção de tecnologias educacionais ${ }^{33}$.

Pesquisas desenvolvidas por enfermeiros, validando cartilhas, apesar de não abordarem o tema da COVID-19, encontraram resultados de IVC semelhantes ao deste estudo e sinalizam para capacidade de tecnologias, validadas, contribuírem para práticas educativas em saúde ${ }^{25,29,32,33}$. Estudos que se dedicaram a apresentar a experiência sobre a produção e divulgação de cartilhas educativas, no contexto da pandemia da COVID-19, afirmam que esta tecnologia possibilita a ampliação, disseminação e acesso a informações seguras, bem como contribuem para estratégias de educação em saúde ${ }^{34-37}$.

Em relação à aparência do material educativo desta pesquisa, que obteve IVC de 1,0 tanto para juízes como para o público-alvo, podemos inferir que houve o reconhecimento pelos participantes de que a cartilha é visualmente atrativa, o que pode despertar o interesse por sua leitura. A utilização de imagens é oportuna por transformar as informações textuais em linguagem visual, facilitando o entendimento dos assuntos tratados e é preciso reconhecer que, em materiais educativos, as figuras ocupam importante papel na comunicação, nesse sentido suas escolhas precisam ser assertivas ${ }^{27}$.
A análise dos dados indicou que, em relação às características que fazem da cartilha um material relevante, foi obtida a meta mínima de concordância estipulada. Esse fato confirma a importância da utilização deste material com vistas a contribuir para a promoção de educação em saúde no contexto escolar, dentro do que este material se propõe. Oferecer informações e orientações, por meio de materiais educativos, pode ser uma importante estratégia para aumentar a adesão às medidas preventivas em relação ao novo coronavírus ${ }^{38}$.

A cartilha foi considerada pertinente quanto à abordagem das etapas que envolvem a limpeza e desinfecção de brinquedos bem como em relação às informações e orientações incorporadas pelo instrumento. É preciso reconhecer que, no atual contexto mundial de pandemia da COVID-19, a produção de informações adequadas, confiáveis e oportunas, tornam-se ainda mais relevante ${ }^{38}$ especialmente por não estarmos vivenciando apenas uma epidemia, mas também uma infodemia ${ }^{32}$. A infodemia caracteriza-se como o excesso de informações, algumas precisas e outras não ${ }^{39}$. Nesse sentido, informações seguras, validadas e baseadas em evidências deve ser considerada uma das respostas que se pode dar em um momento de pandemia.

Mesmo com um IVC superior a 0,8 para quase todos os itens avaliados, incorporamos a maioria das sugestões dos participantes conforme apresentamos nos resultados (Quadro 1). Entretanto, algumas sugestões dos juízes não foram totalmente acatadas na 
Quadro 1. Sugestões, modificações realizadas na cartilha e opinião de juízes e do público-alvo sobre a cartilha.

\begin{tabular}{|c|c|}
\hline Sugestões dos juízes & Modificação realizada \\
\hline \multirow{4}{*}{ Inserir Informações. } & Maior detalhamento sobre a técnica de higienização das mãos com inserção de figura (J1). \\
\hline & $\begin{array}{l}\text { Adicionar outros sintomas da covid-19 como coriza, dor de garganta, cefaleia e anosmia, e que em } \\
\text { crianças podem estar presentes diarreia e manchas vermelhas pelo corpo (J18). }\end{array}$ \\
\hline & Inserir orientações sobre o que fazer em caso de presença de sinais e sintomas da COVID-19 (J6). \\
\hline & Detalhar os cuidados com o uso de máscaras por adultos e crianças (J2). \\
\hline Troca de termos & $\begin{array}{l}\text { Inserimos ao final um "teste seus conhecimentos sobre o novo coronavírus e a limpeza e } \\
\text { desinfecção dos brinquedos" em formato de perguntas, sobre o conteúdo da cartilha (J1). }\end{array}$ \\
\hline \multirow{5}{*}{$\begin{array}{l}\text { Mudança na } \\
\text { aparência }\end{array}$} & Trocamos a palavra "efetivo" para "apropriado" (J9). \\
\hline & A palavra "Alerta" por "Fique atento" (J10). \\
\hline & Uso correto de máscaras por "Uso correto das máscaras (PA2)" \\
\hline & Modificação no estilo/fonte da letra dos títulos das páginas da cartilha (J5). \\
\hline & Evitar o uso de palavras em Caixa alta (J2). \\
\hline Unidade de Sentido & Opinião dos juízes e público-alvo \\
\hline Aparência & Muito bem ilustrado. (J4) Achei muito criativa e chamativa, (PA1) É muito bem ilustrada (PA8). \\
\hline Conteúdo e Clareza & $\begin{array}{l}\text { Objetivos estão claros, textos fáceis de leitura; (PA5) Textos ilustrativos e de fácil compreensão; (J5) } \\
\text { Palavras de fácil entendimento; (J5) Gostei do conteúdo, o material ficou ótimo (J8). }\end{array}$ \\
\hline Relevância & $\begin{array}{l}\text { Acredito que essas medidas devem ser incentivadas e realizadas após a pandemia (J14). } \\
\text { Interessante (PA5); Parabéns pelo trabalho e iniciativa (PA1, PA6, PA4, J1, J9, J14, J19); Excelente } \\
\text { cartilha, poderia ser entregue aos pais pelas escolas (PA8); Gostei muito da cartilha (PA1, PA3); } \\
\text { Adorei o material, espero que seja utilizado nas escolas dos meus filhos (J1); Muito adequado } \\
\text { e necessário (J2); Esse material pode ser direcionado, ainda, para os profissionais de saúde que } \\
\text { trabalham com crianças em brinquedotecas, por exemplo (J19). }\end{array}$ \\
\hline $\begin{array}{l}\text { Aplicabilidade ao } \\
\text { contexto da Pandemia }\end{array}$ & $\begin{array}{l}\text { Utilizaremos também como um documento de estudo no planejamento da escola. (PA3); Minha } \\
\text { avaliação do material é que consegue atender as necessidades das escolas. (PA5); Ajudará até } \\
\text { na elaboração do checklist (protocolo); (PA3) Parabéns pela aplicabilidade do projeto, frente a } \\
\text { pandemia do COVID } 19 \text { (J9). O tema é extremamente importante, principalmente no momento em } \\
\text { que estamos vivendo (J14). Material informativo que possibilitará grande alcance e cuidado diário } \\
\text { ao brincar/aprender/fazer, pois o brincar faz parte da aprendizagem e é uma ação de ocupação da } \\
\text { criança. (J18). }\end{array}$ \\
\hline
\end{tabular}

Fonte: Dados da pesquisa, 2020

* J: codificação para os Juízes seguido de número (1 a 20) atribuído ao questionário respondido por ele. ** PA: Codificação para Público-alvo que seguido de número (1 a 16) atribuído ao questionário respondido por cada um.

cartilha, como, por exemplo, incluir a limpeza e desinfecção dos brinquedos disponíveis no parque infantil como escorregador, casinhas, gangorra entre outros brinquedos como bicicletas e velotrol. Esses brinquedos, em função do tamanho, exigem estratégias diferentes de limpeza e desinfecção, e implicaria em uma cartilha específica para eles. Outra sugestão seria incluir a orientação de limpeza e desinfecção de brinquedos com mais de um tipo de material como tecido, plástico e metal. Nesse caso, como cada material poderá exigir o uso de diferentes desinfetantes $^{12}$, a limpeza e desinfecção poderá ser ineficaz ou danificar o brinquedo, atendendo, assim, parcialmente às sugestões, no final da cartilha inserimos uma dica de como evitar o uso de brinquedos com mais de um tipo de material, e que brinquedos de parques possuem especificidades quanto à sua limpeza e desinfecção, e que não foi objeto de estudo do material produzido.

Os itens referentes à capacidade da cartilha em gerar interesse e motivar a leitura até o final obtiveram IVC no limiar de concordância. Para potencializar esses aspectos, recomendase a utilização da cartilha de forma coletiva, em capacitações, reuniões educativas, mesmo que virtuais, pois a interatividade favorece o processo de ensino-aprendizagem ${ }^{40}$ e, portanto, 
acredita-se que estimule discussões e a assimilação dessas práticas no cotidiano educacional.

Devemos considerar que a elaboração de tecnologias educacionais exige um conjunto de saberes e procedimentos tais como revisão das evidências científicas, valorização da experiência profissional, definição do objetivo do material produzido, seleção do público-alvo, tipo de material, temas, ilustrações e conteúdo de linguagem acessível ${ }^{41}$. Durante o processo de elaboração da cartilha, essas dimensões foram consideradas o que resultou, como pode ser identificado nos resultados apresentados, em um material de qualidade e com validação dos participantes.

Esse processo sinaliza que a cartilha constitui uma tecnologia educacional que pode apoiar práticas de educação em saúde, o que é confirmado por outros estudos ${ }^{19,42,43}$ que se dedicaram a produzir materiais semelhantes. Pesquisas têm revelado que tecnologias educacionais são importantes dispositivos para a medição de processos de ensino e aprendizagem em diferentes espaços de educação continuada ${ }^{40,42}$.

A tecnologia educacional enquanto uma ferramenta que pode ser utilizada dentro do processo de educação em saúde, auxilia na mediação das atividades realizadas pelos enfermeiros, durante o compartilhamento das informações, contudo requer o planejamento de ações que atendam às necessidades do público-alvo para o qual a tecnologia se destina ${ }^{40}$. Nesse sentido, inferimos que o material produzido neste estudo deve ser utilizado pelo enfermeiro, a partir de um planejamento contextualizado para as diferentes realidades escolares que se apresentam no atual cenário de pandemia da COVID-19.

\section{CONCLUSÃO E IMPLICAÇÕES PARA A PRÁTICA}

A cartilha educativa foi validada para seu conteúdo e aparência, e pode ser considerada relevante para contribuir com práticas educativas em escolas infantis, no atual contexto da pandemia da COVID-19.

Concluímos que os resultados desta investigação geraram uma tecnologia educacional de utilidade para escolas, creches e também para a saúde pública, considerando que, no atual contexto de pandemia, há escassez de evidências consistentes sobre o tema e há necessidade de produção de materiais educativos confiáveis e validados. Assim, inferimos que profissionais da educação e enfermeiros poderão fazer o uso dessa tecnologia como instrumento de educação em saúde para prevenção da disseminação da COVID-19, no ambiente escolar.

A cartilha poderá ser utilizada pelo público-alvo como um material de apoio em processos de educação em saúde com a comunidade escolar e também pode ser consultada, pelos profissionais responsáveis pela higienização dos brinquedos, no desenvolvimento da rotina de limpeza e desinfecção desses objetos. Poderá, ainda, ser adotada por enfermeiros no desenvolvimento de práticas de educação em saúde direcionadas aos profissionais das escolas.
É oportuno considerar a possibilidade do material produzido neste estudo contribuir com a elaboração de tecnologias educacionais em outros cenários de atenção à criança como hospitais e brinquedotecas, favorecendo a atuação de brinquedistas.

Este estudo apresenta como limitação a ausência de uma etapa prévia, com pesquisa de campo, para investigar junto à comunidade escolar quais as necessidades desta em relação às estratégias de prevenção ao novo coronavírus envolvendo os brinquedos de uso pedagógico. Reconhecemos também que, apesar de validado pelo público-alvo, este material educativo precisa ser usado em estudos futuros, em escolas, para compreender de forma detalhada a aplicabilidade e eficácia dessa tecnologia educacional.

\section{FINANCIAMENTO}

PIBEX - Programa Institucional de Bolsas de Extensão. Universidade Federal de São João Del Rei - Campus Centro Oeste. Divinópolis - MG, Brasil. № processo associado: Edital 009/2019/PIBEX/2020. Concessão de bolsas do Programa de Extensão "Continuidade do Cuidado às Crianças com Condições Crônicas e suas Famílias.

\section{CONTRIBUIÇÕES DOS AUTORES}

Desenho do estudo. Patrícia Pinto Braga.

Coleta ou produção dos dados. Patrícia Pinto Braga. Dayse

Bazílio Rosa de Souza. Marina Guedes Pinto.

Análise de dados. Patrícia Pinto Braga. Márcia Christina Caetano Romano. Elaine Cristina Rodrigues Gesteira. Verônica Gomes dos Santos. Dayse Bazílio Rosa de Souza. Marina Guedes Pinto.

Interpretação dos resultados. Patrícia Pinto Braga.. Elaine Cristina Rodrigues Gesteira. .Elaine Cristina Rodrigues Gesteira. Verônica Gomes dos Santos. Dayse Bazílio Rosa de Souza. Marina Guedes Pinto.

Redação e revisão crítica do manuscrito. Patrícia Pinto Braga .Elaine Cristina Rodrigues Gesteira. Márcia Christina Caetano Romano. Dayse Bazílio Rosa de Souza. Marina Guedes Pinto. Verônica Gomes dos Santos.

Aprovação da versão final do artigo. Patrícia Pinto Braga .Elaine Cristina Rodrigues Gesteira. Márcia Christina Caetano Romano. Dayse Bazílio Rosa de Souza. Marina Guedes Pinto. Verônica Gomes dos Santos.

Responsabilidade por todos os aspectos do conteúdo e a integridade do artigo publicado. Patrícia Pinto Braga .Elaine Cristina Rodrigues Gesteira. Márcia Christina Caetano Romano. Dayse Bazílio Rosa de Souza. Marina Guedes Pinto. Verônica Gomes dos Santos.

\section{EDITOR ASSOCIADO}

Candida Primo Caniçali https://orcid.org/0000-0001-5141-2898

\section{EDITORA CIENTÍFICA}

Ivone Evangelista Cabral https://orcid.org/0000-0002-1522-9516 


\section{REFERÊNCIAS}

1. World Health Organization. WHO Coronavirus Disease (COVID-19) Dashboard: COVID-19 [Internet]. Geneva:WHO;2020. Disponível em: https://covid19.who.int/

2. Harapan H, Itoh N, Yufika A, Winardi W, Keam S, Te H et al. Coronavirus disease 2019 (COVID-19): a literature review. J Infect Public Health. 2020;13(5):667-73. http://dx.doi.org/10.1016/j.jiph.2020.03.019. PMid:32340833.

3. Ministério da Saúde (BR). Coronavírus (COVID-19) [Internet]. Brasília: Ministério da Saúde;2020 [citado 2020 nov 23]. Disponível em: https:// coronavirus.saude.gov.br/

4. Whittaker E, Bamford A, Kenny J, Kaforou M, Jones CE, Shah P et al. Clinical characteristics of 58 children with a pediatric inflammatory multisystem syndrome temporally associated with SARS-CoV-2. JAMA 2020;324(3):259-69. http://dx.doi.org/10.1001/jama.2020.10369. PMid:32511692.

5. Wu Z, McGoogan JM. Characteristics of and important lessons from the Coronavirus Disease 2019 (COVID-19) Outbreak in China. JAMA 2020;323(13):1239-42. http://dx.doi.org/10.1001/jama.2020.2648. PMid:32091533.

6. Livingston E, Bucher K. Coronavirus Disease 2019 (COVID-19) in Italy. JAMA. 2020;323(14):1335. http://dx.doi.org/10.1001/jama.2020.4344. PMid:32181795.

7. Ministério da Saúde (BR). Boletim Epidemiológico Especial 44. Doença pelo Coronavírus COVID-19. Semana Epidemiológica 53 [Internet] Brasília: Ministério da Saúde; 2020 [citado 2020 nov 23]. Disponível em: https://www.gov.br/saude/pt-br/media/pdf/2021/janeiro/07/ boletim_epidemiologico_covid_44.pdf

8. Ministério da Saúde (BR). Boletim Epidemiológico Especial 52. Doença pelo Coronavírus COVID-19. Semana Epidemiológica 8 [Internet] Brasília: Ministério da Saúde; 2021 [citado 2020 nov 23]. Disponível em: https://www.gov.br/saude/pt-br/media/pdf/2021/marco/05/ boletim_epidemiologico_covid_52_final2.pdf

9. Nota técnica GVIMS/GGTES/ANVISA № 04/2020 (BR). Orientações para serviços de saúde: medidas de prevenção e controle que devem ser adotados durante a assistência aos casos suspeitos ou confirmados de infecção pelo novo Coronavírus (SARS-CoV-2). Diário Oficial da União [periódico de Internet], Brasília (DF), 31 mar. 2020 [citado 2020 nov 23]. Disponível em: https://www.telessaude.unifesp.br/images/ downloads/Nota\%20Te\%CC\%81 cnica\%20n\%2004-2020\%20GVIMSGGTES-ANVISA-ATUALIZADA.pdf

10. Vilelas JMS. The new coronavirus and the risk to children's health. Rev Latino-Am. Enfermagem. 2020;28:e3320. http://dx.doi.org/10.1590/15188345.0000.3320.

11. Cao Q, Chen YC, Chen CL, Chiu CH. SARS-CoV-2 infection in children: transmission dynamics and clinical characteristics. J Formos Med Assoc. 2020;119(3):670-3. http://dx.doi.org/10.1016/j.jfma.2020.02.009. PMid:32139299.

12. Kampf G, Todt D, Pfaender S, Steinmann E. Persistence of coronaviruses on inanimate surfaces and their inactivation with biocidal agents. J Hosp Infect.2020;104(3):246-51. http://dx.doi.org/10.1016/j.jhin.2020.01.022.

13. Moriarty LF, Plucinski MM, Marston BJ, Kurbatova EV, Knust B, Murray EL et al. Public Health Responses to COVID-19 Outbreaks on Cruise Ships - Worldwide, February-March 2020. MMWR Morb Mortal Wkly Rep. 2020;69(12):347-52. http://dx.doi.org/10.15585/mmwr.mm6912e3. PMid:32214086.

14. Carvalho AS, Cunha FL, Hofling RTB, Imparato JCP, Fantinato V. Métodos de desinfecção de brinquedos em consultórios odontológicos. Rev Gaucha Odontol. [Internet]. 2004 [citado 2020 nov 23];52(3):1658. Disponível em: http://www.revistargo.com.br/include/getdoc. php?id $=651$ \&article $=295 \&$ mode $=$ pdf

15. Jimenez $\mathrm{M}$, Martinez $\mathrm{Cl}$, Chaidez $\mathrm{C}$. Disinfection alternatives for contact surfaces and toys at child care centers. Int $\mathrm{J}$ Environ Health Res. 2010;20(6):387-94. http://dx.doi.org/10.1080/09603123.2010.49 1851. PMid:21161800.

16. Furquim FC, Medina LT. Identificação de Staphylococcuse enterobactérias em brinquedos de uma creche em Mato Grosso, Brasil. J Health Sci. 2015;17(3):181-8. http://dx.doi.org/10.17921/2447-8938.2015v17n3p\%25p.
17. Davis SG, Corbitt AM, Everton VM, Grano CA, Kiefner PA, Wilson AS et al. Are ball pits the play-ground for potentially harmful bacteria? Pediatr Nurs. 1999;25(2):151-5. PMid:10532011.

18. De Paula NM, Costa E. Brinquedoteca hospitalar e a importância da higienização dos brinquedos. SCIAS - Arte/Educação [Internet]. 2014 [citado 2020 nov 23];3(3):51-66. Disponível em: https://revista.uemg. br/index.php/scias/article/view/589

19. Sabino LMM, Ferreira AMV, Joventino ES, Lima FET, Penha JC, Lima KF et al. Elaboração e validação de cartilha para prevenção da diarreia infantil. Acta Paul Enferm. 2018;31(3):233-9. http://dx.doi. org/10.1590/1982-0194201800034.

20. Lemos M, Fontoura M. A integração da educação e trabalho na saúde e a Política de Educação Permanente em Saúde do SUS-BA. Rev Baiana Saúd Púb. 2009;33(1):113-20. http://dx.doi.org/10.22278/23182660.2009.v33.n1.a195.

21. Blasi DG, El Dib RP, Correa I. Efetividade da higienização de brinquedos infantis na redução microbiana: revisão sistemática da literatura. Vigil. Sanit. Debate. 2016;4(3):96-102. https://dx.doi.org/10.22239/2317269x.00723.

22. Polit DF, Beck CT. Fundamentos de pesquisa em enfermagem - Avaliação de evidências para a prática da enfermagem [Internet]. 7. ed. Porto Alegre: Artmed;2011 [citado 2020 nov 23]. 669 p. Disponível em: https:// books.google.com.br/books?hl=ptBR\&lr=\&id=2AKpDAAAQBAJ

23. Pasquali L. Psicometria: teoria e aplicações [Internet]. Brasília: Editora Universidade de Brasília; 1997 [citado 2020 nov 23]. 289 p. Disponível em: https://docero.com.br/doc/x0n50n

24. Melo RP, Moreira RP, Fontenele FC, Aguiar ASC, Joventino ES, Carvalho EC. Critérios de seleção de experts para estudos de validação de fenômenos de enfermagem. Rev Rene. [Internet]. 2011 [citado 2020 nov 23];12(2):424-31. Disponível em: http://periodicos.ufc.br/rene/ article/view/4254/3285

25. Alexandre NMC, Coluci MZO. Validade de conteúdo nos processos de construção e adaptação de instrumentos de medidas. Cien Saude Colet. 2011;16(7):3061-8. http://dx.doi.org/10.1590/S1413-81232011000800006. PMid:21808894.

26. Lima MB, Rebouças CBA, Castro RCMB, Cipriano MAB, Cardoso MVLML, Almeida PC. Construction and validation of educational video for the guidance of parents of children regarding clean intermittent catheterization. Rev Esc Enferm USP. 2017;51:e03273. http://dx.doi. org/10.1590/S1980-220X2016005603273. PMid:29267731.

27. Sena JF, Silva IP, Lucena SKP, Oliveira ACS, Costa IKF. Validação de material educativo para o cuidado da pessoa com estomia intestinal. Rev Lat Am Enfermagem. 2020;28:e3269. http://dx.doi.org/10.1590/15188345.3179.3269. PMid:32401899.

28. Canva 8 . [Internet]. [citado 2020 nov 23]. Disponível em: https://www. canva.com/pt_br

29. Centers for Disease Control and Prevention. How to clean and disinfect schools to help slow the spread of Flu [Internet]. USA: CDC; 2018 [citado 2020 nov 23]. Disponível em: https://www.cdc.gov/flu/school/cleaning. $\mathrm{htm}$

30. Cruz FOAM, Ferreira EB, Vasques Cl, Mata LRF, Reis PED. Validation of an educative manual for patients with head and neck cancer submitted to radiation therapy. Rev Lat Am Enfermagem. 2016;24(0):e2706. http:// dx.doi.org/10.1590/1518-8345.0949.2706. PMid:27305178.

31. Moura IH, Silva AFR, Rocha AESH, Lima LHO, Moreira TMM, Silva ARV Construction and validation of educational materials for the prevention of metabolic syndrome in adolescents. Rev Lat Am Enfermagem. 2017;25(0):e2934. http://dx.doi.org/10.1590/1518-8345.2024.2934. PMid:29020125.

32. Organização Pan-Americana de Saúde. Entenda a infodemia e a desinformação na luta contra a COVID-19 [Internet]. Washington: Departamento de Evidência e Inteligência para Ação em Saúde/ OPAS; 2020 [citado 2020 nov 23]. 5 p. Disponível em: https://iris. paho.org/bitstream/handle/10665.2/52054/Factsheet-Infodemic_por. pdf? sequence $=14$

33. Alexandre NMC, Coluci MZO. Validade de conteúdo nos processos de construção e adaptação de instrumentos de medidas. Cien Saude Colet. 2011 jul;16(7):3061-8. http://dx.doi.org/10.1590/S141381232011000800006. PMid:21808894. 
34. Rodrigues LN, Santos AS, Gomes PPS, Silva WCP, Chaves EM Construction and validation of an educational booklet on care for children with gastrostomy. Rev Bras Enferm. 2020;73(3):e20190108. http://dx.doi.org/10.1590/0034-7167-2019-0108. PMid:32321143.

35. Cruz FOAM, Ferreira EB, Vasques CI, Mata LRF, Reis PED. Validation of na educative manual for patients with head and neck câncer to radiation therapy. Rev Lat Am Enfermagem. 2016;24(0):e2706. http:// dx.doi.org/10.1590/1518-8345.0949.2706. PMid:27305178.

36. Silva RCR, Raimundo ACL, Santos CTO, Vieira ACS. Construção de cartilha educativa sobre cuidados com crianças frente à pandemia COVID-19: relato de experiência. Rev Baiana Enferm. 2020;34:e37173. http://dx.doi.org/10.18471/rbe.v34.37173.

37. Gouveia AO, Silva HRS, Batista JBS No. Saúde mental em tempos de pandemia de Covid-19: construção de cartilha educativa com orientações para o período de pandemia. Enferm. Foco (Brasília). [Internet]. 2020 [citado 2020 nov 23];11(1, n. esp):168-73. Disponível em: http://revista. cofen.gov.br/index.php/enfermagem/article/view/3600/821

38. Coutinho JG, Padilla M. Informação adequada, confiável e oportuna em tempos de pandemia de COVID-19. Rev Panam Salud Publica. 2020;44:e118. http://dx.doi.org/10.26633/RPSP.2020.118. PMid:32994790.
39. Zarocostas J. How to fight an infodemic. Lancet. 2020;395(10225):676. http://dx.doi.org/10.1016/S0140-6736(20)30461-X. PMid:32113495.

40. Rvachew S, Thompson D, Dey R. Can technology help close the gender gap in literacy achievement? Evidence from boys and girls sharing eBooks. Int J Speech Lang Pathol. 2020;22(3):290-301. http://dx.doi. org/10.1080/17549507.2019.1692905. PMid:31771365.

41. Teixeira E, Medeiros HP, Nascimento MHM. Referenciais metodológicos para validação de tecnologias cuidativo-educacionais. In: Nietsche EA, Teixeira E, Medeiros HP, organizadores. Tecnologias cuidativoeducacionais: uma possibilidade para o empoderamento do(a) enfermeiro(a). Porto Alegre: Moriá; 2014. p. 113-27.

42. Wild CF, Nietsche EA, Salbego C, Teixeira E, Favero NB. Validation of educational booklet: an educational technology in dengue prevention. Rev Bras Enferm. 2019 set 16;72(5):1318-25. http://dx.doi.org/10.1590/00347167-2018-0771. PMid:31531657.

43. Khurana S, Rao BK, Lewis LES, Bhat R, Purkayastha J, Kamath A et al Development and validation of educational leaflet for caregivers of preterm infants. J Clin Diagn Res. 2016;10(7):1-4. http://dx.doi.org/10.7860/ JCDR/2016/19206.8109. PMid:27630939. 